\title{
Mini-incision Hemithyroidectomy-Incision Closure with Subcuticular Suture Versus No Suture of Subcutaneous Tissue and Skin
}

\author{
Sade Rajkumar, Vaidyula Sudha Spandana, Mutya Subrahmanyam* \\ Department of General Surgery, Kamineni Institute of Medical Sciences, Narketpally, Telangana, India
}

Email address:

subrahmanyam_mutya@yahoo.com (M. Subrahmanyam)

${ }^{*}$ Corresponding author

To cite this article:

Sade Rajkumar, Vaidyula Sudha Spandana, Mutya Subrahmanyam. Mini-incision Hemithyroidectomy-Incision Closure with Subcuticular Suture Versus No Suture of Subcutaneous Tissue and Skin. Journal of Surgery. Vol. 7, No. 5, 2019, pp. 123-127.

doi: $10.11648 /$ j.js.20190705.12

Received: July 7, 2019; Accepted: August 6, 2019; Published: August 23, 2019

\begin{abstract}
Minimally invasive thyroid surgery techniques (MIT) have the advantage of reduction of tissue trauma, early hospital discharge, and better neck wound cosmetic appearance, while maintaining the same surgical outcome as traditional thyroidectomy. In addition to MIT, methods of skin closure contribute to the overall aesthetic outcome and patient's satisfaction. Methods of skin closure depends largely on the surgeon's choice based upon the need for a rapid, economic, and reproducible technique. The aim of the study is to compare the quality of resulting scar at 6 weeks after mini-incision hemithyroidectomy with subcuticular absorbable sutures or no subcuticular or skin sutures for the closure of the incision. Methods. There are 102 patients undergoing mini-incision hemithyroidectomy. Fifty two patients were randomly assigned to closure with approximation of subcutaneous tissue and skin by bringing the wound edges together by pressing with forceps and the other 50 with subcuticular absorbable suture. Post-operatively patients were assessed for pain and it was observed that pain was less in group treated without skin or subcutaneous sutures. At 6 weeks, the scar was evaluated by the patient after viewing in the mirror and graded as 1-5. Results. Pain during the postoperative period was significantly less in patients without subcutaneous or skin sutures. Assessment of scar appearance by the patients showed a statistically significant difference in favor of no skin or subcutaneous suture $(n=48)$ as compared to subcuticular suture $(n=40)$. Conclusion. Incision closure of mini -incision hemithyroidectomy without subcuticular or skin sutures and only by approximation had less pain postoperatively as compared to subcuticular suture. Scar assessment by patients revealed better satisfaction by this method.
\end{abstract}

Keywords: Skin Closure, Mini-incision Thyroidectomy, Scar Appearance

\section{Introduction}

Scar remains the only evidence of the surgeon's skill and not infrequently all his efforts are judged on its final appearance. Since times immemorial, surgeons have strived to produce "invisible scars". The skin closure is generally done with sutures which provide an extra source of contamination and are a potential source of foreign body reaction in the susceptible subcutaneous tissue and also time consuming during application. Over the time, wound closure techniques have evolved from the earliest suturing materials to resources that include synthetic absorbable sutures, staples, tapes, and adhesive compounds. The ideal method of incision closure should be simple, safe, rapid, inexpensive, painless, bactericidal, and result in optimal cosmetic appearance of the scar. Sub cuticular suture is often considered to be a technique which requires a particular surgeon's expertise and a longer time of execution, even if it has been indicated as the gold standard for neck surgery [1].

Thyroidectomy is a surgery commonly performed across the world. The incision used for thyroid surgery has become shorter overtime, from the classical $10 \mathrm{~cm}$ long Kocher incision to the shortest $15 \mathrm{~mm}$ access achieved with minimally invasive open or video-assisted thyroidectomy. This is mainly to achieve a good cosmetic outcome along with less postoperative morbidity. The aesthetic outcome is 
particularly relevant in thyroid surgery since patients are mostly women and young adults and since the incision is in a highly sensitive and in visible anatomic location. Cosmetic concern about the final scar appearance contributed to motivate the development of minimally invasive approaches for thyroid surgery and parathyroid surgery over the last decade [2-13]. In addition to MIT, methods of skin closure play a role in the cosmetic outcome of surgery.

We tried to compare closure of wound with absorbable subcuticular suture and without closure of skin and subcutaneous tissue by bringing the edges of the wound together and maintain the position by gently pressing with fingers and by applying gauze dressing.

\section{Material and Methods}

A series of consecutive patients undergoing hemithyroidectomy from January 2013 to March 2016 formed material for this study. The only inclusion criterion was being a candidate to a hemithyroidectomy, regardless of the diagnosis. The exclusion criteria were previous surgery of thyroid. The operation was performed through a minimal open cervical incision, as described earlier [2]. Surgery was performed by the same surgeon (MS). The incision length was between 1.5 and $3 \mathrm{~cm}($ mean $=2.13 \pm 0.79)$. Meticulous hemostasis was achieved before approximation and closure of skin layers. Skin closure was applied after reapproximating the strap muscles of the neck and closing platysma with absorbable suture material. In both the groups the wound was closed with a gauze dressing. In group 1 (52 patients), the subcutaneous tissue and the skin of both sides were approximated with fingers and a toothed forceps was used to keep them pressed and maintain the position. In the group 2 (50), subcuticular sutures were applied with absorbable 3. 0 suture material. After the operation, patients received a standard postoperative protocol and analgesic regime. Post-operative pain assessment was done using visual analogue scale ranging from 1-10 as described earlier [2]. The assessment of the scar was done after 6 weeks in both the groups. The patient was asked to stand in front of a mirror, and mention about the satisfaction of the scar. The scar was graded as per patients satisfaction as 5 grades from 0 to 5,5 being complete satisfaction and 0 being dissatisfied. The results were analysed using chi-square test.

Enrolled patients were randomly assigned through a series of random numbers through the picking up of chits. Institutional ethical committee has approved the study. Written informed consent was obtained from all the patients.

\section{Results}

Of the 52 patients in group 1,42 were women and 10 men. The age ranged from 18 to 62 . In group 2, 43 were women and 17 men. The duration of symptoms ranged from 1 to 20 years in group 1 and 2 to $18 \mathrm{yrs}$, in group 2 . The size of the neck swelling ranged from 2 t0 $4 \mathrm{cms}$ in group 1 and 1.5 to 3.5 in group 2. In group I, the operation time was 25 mins. with a range of 20-35 mins, where as in group 2, it was 40 mins (range 30-48 mins,). Hemithyroidectomy was done in all patients in both groups. There was no bleeding in patients of either group. In the post operative period, there was no bleeding or any other complication. Table 1 shows patient details.

Post-operative pain assessment showed in42 patients, the pain is minimum ranging from $1-5$, in 8 , it was $5-10$ in group1, where as in group 2, 38 patients had pain ranging from (1-5) and 12 patients had pain ranging from (5-10, $\mathrm{p}<$ 0.05 , significant). The wounds healed in all patients with out any complications. The scar assessment done at 6 weeks as described by the patients showed excellent in 44, good in 6 , average in 2 as per the patients's assessment. In group 2, the assessment of scar was excellent in 36 , good in 10 and average in $4 .(p<0.05)$. Figure 1 shows approximation of incision after hemothyroidectomy with forceps. Figure 2 shows the scar in the study group after 6 weeks.

Table 1. Patients detais.

\begin{tabular}{lll}
\hline Details & Group 1 (n=52) & Group 2 (n=50) \\
\hline Males & 10 & 17 \\
Females & 42 & 43 \\
Duration of symptoms & $1-20 \mathrm{yrs}$ & $2-18 \mathrm{yrs}$ \\
Size of swelliong & $2-4 \mathrm{~cm}$ & $1.5-3.5 \mathrm{~cm}$ \\
Diagnosis & & \\
Adenoma & 42 & 44 \\
Cyst & 10 & 8 \\
Surgery time & & \\
(Mean) & $25 \mathrm{~min}$ & $40 \mathrm{~min}$ \\
(Range) & $20-35 \mathrm{~min}$ & $30-48 \mathrm{~min}$ \\
\hline
\end{tabular}

Table 2. Scar assessment by the patients.

\begin{tabular}{lll}
\hline Result & $\begin{array}{l}\text { No. of patients } \\
\text { Group 1 }(\mathbf{n = 5 2})\end{array}$ & $\begin{array}{l}\text { NO. of patients } \\
\text { Group 2 }(\mathbf{n}=\mathbf{5 0})\end{array}$ \\
\hline Excellent & 44 & 36 \\
Good & 8 & 10 \\
Average & 2 & 4 \\
\hline
\end{tabular}

$(p<0.05$, significant $)$

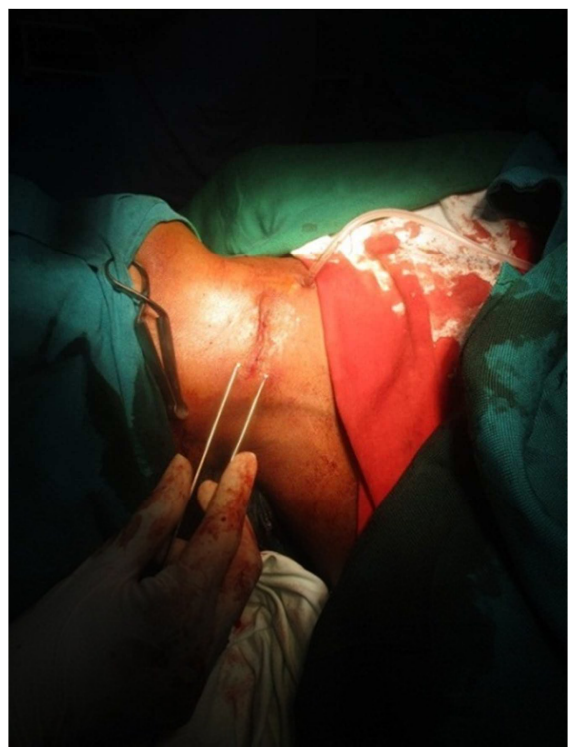

Figure 1. Incision being approximated with forceps. 


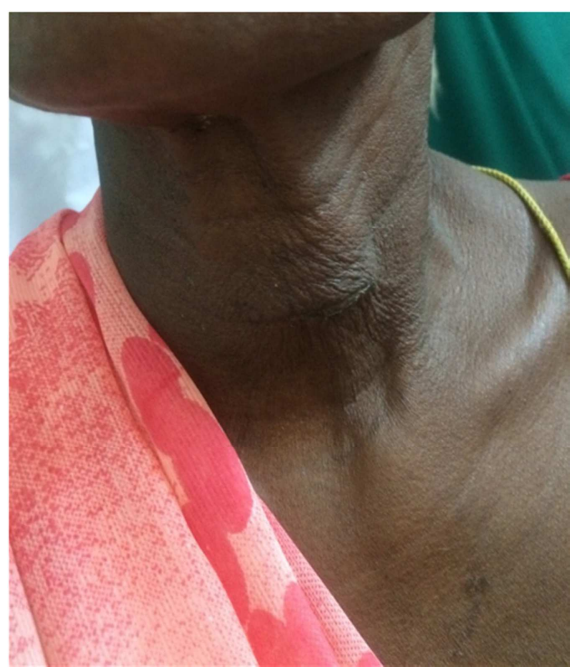

Figure 2. Scar 6 weeks after hemithyroidectomy without subcutaneous and skin closure.

\section{Discussion}

The concept of minimal access surgery is not new and it has been practiced in many other surgical specialties for over two decades now. It is attractive because patients are concerned not only about the results of treating their thyroid disease, but also outcomes such as better cosmesis, reduced hospital stay and decreased pain. The three most commonly utilized approaches for thyroid surgery are minimally invasive video-assisted thyroidectomy (MIVAT), minimal invasive thyroid surgery through a direct lateral mini-incision approach (MITS) or a central mini incision approach or an incision at the upper pole of the swelling [2]. If skin suturing techniques are improved, the results will be more encouraging.

In thyroid surgery, aesthetic outcome is particularly relevant since patients are mostly women and young adults and since the incision is in a highly sensitive and visible anatomic location. Development of minimally invasive approaches for thyroid surgery and parathyroid surgery over the last decade have been motivated by the cosmetic concern about the final appearance of the scar. [1-3]. Minimally invasive thyroid surgery techniques (MIT) are different but all share the same goals: reduction of tissue trauma, early hospital discharge, and better neck wound cosmetic appearance, while maintaining the same surgical outcome as traditional thyroidectomy [4].

In addition to MIT, methods of skin closure contribute to the overall aesthetic outcome and patient's satisfaction. Methods of skin closure vary in published series and are largely the results of surgeon's choice based upon the need for a rapid, economic, and reproducible technique [5].

Skin closure techniques include the use of the tissue adhesive octyl-cyanoacrylate, introduced 15 years ago in clinical practice as an ideal system of wound closure [6].

Many studies showed that tissue adhesive is an acceptable alternative to standard wound closure since it yields similar clinical and aesthetic results.
Furthermore, a recent study comparing adhesive strips and subcuticular absorbable suture in neck incision found an equal overall wound appearance after 6 weeks [13]. On the contrary, an Italian study [14] showed a worse aesthetic result after thyroidectomy for the tissue adhesive group.

Tissue adhesive was advocated to decrease pain and discomfort, since many patients are anxious at the prospect of removal of sutures and occasionally describe it as of more concern than the procedure itself. This aspect can influence patient's overall satisfaction. When considering a cervical incision, the aesthetic outcome is considered highly significant, since the wound is almost permanently on view. This aspect becomes furthermore important if we consider that young women constitute a large proportion of patients affected by a thyroid disease. In recent years, surgeons have become increasingly interested in obtaining an optimal aesthetic outcome. Minimally invasive thyroidectomy techniques have been developed in an effort to improve aesthetic results as well as minimizing pain and shortening hospital stay. A shorter incision, however, does not necessarily confer an improvement of patient overall satisfaction and opinion on aesthetic outcome. In this context, the choice of the method of suture can be a critical factor in the scar appearance. Many of previous studies comparing tissue adhesive with other techniques in neck surgery relied on a simple mono-dimensional numeric scale of measure of patient's satisfaction $[5,10,14]$. We also used patient's satisfaction as the measure of aesthetic outcome.

Although most facial wounds heal without complications, owing to the abundant blood supply of the region, mismanagement may result in infection, wound dehiscence, and unsightly and dysfunctional scar. Long term aesthetic appearance of the scar is one of the most important outcomes of wound repair. The aim of thyroid surgeries is to achieve the healing with no discharge or infection, minimal oedema and scarring. [8]

Having good vascular supply, neck incisions heal very quickly, allowing to remove the sutures early. It is earlier than usual for removal of sutures but had shown success from the final cosmetic appearance with visual linear analogue scores. [9]

When considering a cervical incision, the aesthetic outcome is considered highly significant, since the wound is almost permanently on view. This aspect becomes furthermore important if we consider that young women constitute a large proportion of patients affected by a thyroid disease. In recent years, surgeons have become increasingly interested in obtaining an optimal aesthetic outcome.

Minimally invasive thyroidectomy techniques have been developed in an effort to improve aesthetic results as well as minimizing pain and shortening hospital stay. A shorter incision, however, does not necessarily confer an improvement of patient overall satisfaction and opinion on aesthetic outcome. In this context, the choice of the method of suture can be a critical factor in the scar appearance.

The choice of wound closure method and material will finally depend on the surgeon's preference. However, this 
study does show that after closing the platysma, approximation of subcutaneous tissue and skin is a good alternative to keep edges together and result in good healing with good cosmetic result. This results in less pain, acceptable neck mobility, excellent scar appearance as compared to subcuticular sutures.

Subcuticular suture is often considered to be a technique which requires a particular surgeon's expertise and a longer time of execution, even if it has been indicated as the gold standard for neck surgery. A recent systematic review could not find difference in the duration of the procedure, time spent on applying subcuticular sutures [1].

It is noteworthy that while the latter two are directly related to the esthetic outcome, pain is a general symptom and for a patient may be difficult to discriminate if pain comes from the scar of the underlying site of the operation (muscles and fascial layer).

Many factors contribute to the determination of patient's satisfaction with surgery related to cultural context and outcome as well as personal factors, like the expectation and the familiarity with what a surgeon would rate as a "normal" outcome. Setting a good communication with the patients and their families and allowing to share a feasible expected outcome could probably be as important as achieving a smaller access and better scars. Since many patients are anxious at the prospect of removal of sutures and occasionally describe it as of more concern than the procedure itself. In most of the studies follow up is limited to 6 weeks, but it is known that the appearance and the assessment of scars tend to get better as time goes on [15-24].

\section{Conclusion}

This study demonstrated that the approximation of subcutaneous and dermal margins has a better cosmetic outcome than the use of absorbable subcuticular sutures,. This observation in association with the favorable rating of patients led us to consider this as the standard method of closure and prompted us to continue following this procedure.

\section{References}

[1] Nagpal BM, KumR G, Nagi GS, Singh P. Sutureless closure of operative skin wounds. MJAFI. 2004, 60: 131-33.

[2] Subrahmanyam M, Sirisha R, Deepthi A, Mishra R. N. Minimally Invasive Hemithyroidectomy Using a Mini Incision over the Upper Pole of Thyroid Swelling. Journal of Surgery. 2015: 3, 21-25.

[3] Kazi R, Katna R, Dwivedi RC, Minimal access thyroid surgery- a new dawn? Ann R Coll Surg Engl 2010; 92: 3612.

[4] Fik Z, Astl J, Zabrodsky M, et al. Minimally invasive videoassisted versus minimally invasive non endoscopic thyroidectomy, Biomed Research International 2014, Article ID 450170, 7 pages, 2014. doi: 10.1155/2014/450170.
[5] Gagner M. Endoscopic subtotal parathyroidectomy in patients with primary hyperparathyroidism, British Journal of Surgery: 1996, 83: 875.

[6] Miccoli P, Berti P, Conte, M et al., Minimally invasive surgery for thyroid small nodules: preliminary report, Journal of Endocrinological Investigation, 1999, 22: 849851.

[7] MiccoliP, Berti P, Frustaci GL et al. Video-assisted thyroidectomy: indications and results. Langenbeck's Archieves of Surgery, 2006, 39: 168-71.

[8] Ferzli GS, Sayad P. Abdo Z et al. Minimally invasive, nonendoscopic thyroid surgery. Journal of the American College of Surgeons. 2001, 192: 665-8.

[9] Norman J, Chheda H, Farrell C. Minimally invasive parathyroidectomy for primary hyperparathyroidism: decreasing operation time and potential complications while improving cosmetic result, The Americ Surgeon, 1998, 64: 391-96.

[10] Touzopoulos P, Karanikas M, ZarogoulidisP, et al. Current surgical status of thyroid diseases, Journal of Multidisciplinary Health care, 2011, 4: 441-49.

[11] Autorino R,. White W, M, Gettman M. T. et al., "Public perception of, "scarless" surgery: a critical analysis of the literature,” Urology, 2012 80: 495-502.

[12] Raklyar E Zloty D. M. Use of a patient and observer scar assessment scale to evaluate the V-Y advancement flap for reconstruction of medial cheek defects. Dermatologic Surgery, 2012, 38. 1968-1974.

[13] Pronio A, di Filippo A, Narilli Pet al., "Closure of cutaneous incision after thyroid surgery: a comparison between metal clips and cutaneous octyl-2-cyanoacrylate adhesive. A prospective randomized clinical trial," European Journal of Plastic Surgery, 2010. 103-110.

[14] A. G. Huppelschoten, J. C. van Ginderen, K. C. van den Broek, A. E. Bouwma, and H. P. Oosterbaan, "Different ways of subcutaneous tissue and skin closure at cesarean section: a randomized clinical trial on the long-term cosmetic outcome," Acta Obstetricia et Gynecologica Scandinavica, 2013, 92: 916-924.

[15] ShirleyE. D., J. O. SandersJ. O. Patient satisfaction: implications and predictors of success," The Journal of Bone \& Joint taneous tissue and skin closure at cesarean section: a randomized clinical trial on the long-term cosmetic outcome. Acta Obstetricia et Gynecologica Scandinavica, 2013, 92: 916-924.

[16] H. Y. Kim, J. W. Kim, J. H. Park, J. H. Kim, and Y. S. Han, "Personal factors that affect the satisfaction of female patients undergoing esthetic suture after typical thyroidectomy," Archives of Plastic Surgery, 201 32: 414-424.

[17] D. Linos, K. P. Economopoulos, A. Kiriakopoulos, E. Linos, and A. Petralias, "Scar perceptions after thyroid and parathyroid surgery: comparison of minimal and conventional approaches," Surgery, 2013, 153: 400-407.

[18] LombardiC. P, R. Bracaglia R. Revelli Let al., "Aesthetic result of thyroidectomy: evaluation of different kinds of skin suture," Annali Italiani di Chirurgia, 2011. 82. 449456. 
[19] Draaijers L. J, Tempelman, T. R. H. BotmanY. A. M et al., "The patient and observer scar assessment: a reliable and feasible tool for scar evaluation," Plastic and Reconstructive Surgery, 2004, 113. 1960-1965.

[20] Consorti F, Milazzo, M. Notarangelo, L. Scardella, and A. Antonaci, "Factors influencing the length of the incision and the operating time for total thyroidectomy," BMC Surgery, 2012. 12: 15-18.

[21] Sakr MF, El-Waghi HF, Zayed AF. Mini incisionthyroidectomy technique: impact of volume of the thyroid gland. Biolife 2017, 5: 518-23.
[22] Noori MF. Open minimal-incision thyroidectomy: Clinical experience with 22 patients and literature review. B as J Surg, 2016, 6: 71-8.

[23] Deliro P, Viani L, MontanaCM, CozzaniF SianesiM. Minimallyinvasive 22. Noori IF. Open mini-incision thyroidectomy; A ten year experience Gland Surg 2016, 5; 295-99.

[24] Dordea M, Aspinal SR, Short and long term cosmesis of cervical thyroidectomy scars, Ann R Coll Surg Eng 2016, 98: 11-17. 